\title{
Top 25 cited articles on Gamma Knife surgery published since 2005 in journals of the American Association of Neurological Surgeons
}

\author{
Veronica L. S. Chiang, MD, ${ }^{1}$ and Jonathan P. S. Knisely, MD² \\ 1Department of Neurosurgery, Yale University School of Medicine, and Yale Cancer Center, New Haven, Connecticut; and \\ 'Department of Radiation Medicine, Hofstra University North Shore-LIJ School of Medicine, Hofstra University, Manhasset, \\ New York
}

http://thejns.org/doi/abs/10.3171/2015.12.JNSLekselllntro

$\mathrm{R}$ adiosurgery can no longer be considered a novel therapeutic approach. It is used across the world in all developed countries as well as in many developing countries. The 25 publications included in this collection span the globe-from North America (the United States and Canada) to Western Europe (Belgium, France, and Germany) and on to Eastern Europe (Czech Republic and Slovakia) and Asia (China and Japan). Radiosurgery is now routinely performed for the definitive treatment of many neurosurgical diseases, including vascular malformations and benign and malignant tumors, as well as functional disorders; it has become a standard-of-care adjunct to surgery in the quest to decrease morbidity for patients with these conditions. This collection of 25 papers is a reflection of the Leksell Gamma Knife Society's efforts to establish the standards by which radiosurgery should be practiced, disseminate the results that would be expected from excellent radiosurgical care, and highlight the remaining questions that need to be addressed to further improve outcomes.

While the use of radiosurgery in functional disorders remains the most debated topic historically, the indication that today sparks the most widespread discussion is the burgeoning trend for using radiosurgery for the treatment of brain metastases. Literature on this topic, while robust and prolific, remains young compared with the articles that follow in this collection. A mere decade ago, medical oncologists saw cancer as a systemic problem, with brain metastases defining the end game for a cancer patient because the tumor was beyond the blood-brain barrier and largely not responsive to systemic treatments. This nihilistic approach towards brain metastases was mirrored by a similar palliative approach, from the perspective of radiation oncology, towards whole brain radiation therapy
(WBRT) - a procedure that has never been decisively shown to affect survival for these patients, but which is a safe, gentle, fractionated approach to minimizing neurological compromise until the patient's inevitable shortterm death. But much has changed since then.

The survival of cancer patients with brain metastases has significantly improved due to the development of targeted therapy and immunotherapy. In addition, technological leaps forward in imaging have facilitated the easy and early detection of brain metastases that are small and asymptomatic, and medical oncologists have recognized the need for screening cranial MR imaging in patients whose tumors have a propensity to spread to the brain. Finally, our pioneer Gamma Knife radiosurgeons have worked hard not only to convince physicians in our own specialty, but also physicians in other specialties and health insurance providers that radiosurgery has a role for brain metastases beyond salvage after WBRT. In fact, radiosurgery is now the recommended first-line treatment for patients who first present with small-volume oligometastatic brain metastases, as recommended by the American Society for Radiation Oncology, the National Cancer Institute's National Comprehensive Cancer Network, and other organizations.

The use of radiosurgery for the treatment of brain metastases is about to escalate exponentially, not only because this makes sense to us as physicians, but also because the consumer market now demands that the use of radiosurgery be based both on a better recognition of the neurocognitive sequelae of WBRT in long-term survivors of all ages and on Class I data from randomized controlled clinical studies that show that definitive radiosurgery without WBRT can improve survival prospects for patients 50 years of age and younger. In addition, the concept of radio- 
surgery not just for the brain but also for cancers in other parts of the body, such as lung, liver, spine, and prostate, is gaining popularity. Consumer demand has resulted in significant growth in the development of advanced-generation radiosurgically capable machines, including the Leksell Gamma Knife Icon from Elekta AB, which allow targeting of extracranial sites, dose fractionation, and frameless treatments.

The rising importance of intracranial metastatic disease in overall cancer management leads to easy predictions of future upheavals in management approaches beyond what has been seen to date. Regional and systemic therapies, such as hippocampal-avoidance WBRT, and drugs, such as memantine hydrochloride to attenuate the impact of WBRT on neurocognition and quality of life, are currently being explored in National Cancer Institute-supported clinical trials. Importantly, single-isocenter linear accelerator-based radiosurgical treatment is gaining interest among many caregivers because of the relative ease and speed that this approach provides for the treatment of multiple intracranial metastatic foci. There will likely be many more patients treated with these approaches in the future, but the equivalence of these new approaches to what has been shown to be possible with Gamma Knife surgery has yet to be demonstrated.

These are exciting technological times. With all of the new radiosurgical machines, it is essential that the radiosurgeon continue to maintain, in the treatment of the brain, the same standards that have brought us to this point. These should include submillimetric targeting accuracy, rapid dose falloff, ease and safety of new lesion planning to minimize high dose overlap, and, ultimately, radiosurgical efficacy and the role of radiosurgery in conjunction with systemic therapies in the whole cancer patient. The development of lasers and focused ultrasound in the treatment of brain lesions is also gaining popularity, and many of these newer tools are being compared to radiosurgery as their standard. As radiosurgeons, we need to ensure that our literature in this area continues to reflect the highest standards for patient care, as do the following 25 mostcited papers since 2005 . 